\title{
Serum level of eight cytokines in Han Chinese patients with systemic lupus erythematosus using multiplex fluorescent microsphere method
}

\author{
ZHIHUA YIN ${ }^{1 *}$, JINXIAN HUANG ${ }^{1,2 *}$, WEIZHEN HE ${ }^{l}$, ZHIJUN CAO ${ }^{l}$, XIUXIA LUO ${ }^{l}$, \\ CHUNRONG ZHANG ${ }^{l}$, ZHIZHONG YE ${ }^{l}$
}

${ }^{1}$ Rheumatology Department, The Affiliated Futian Hospital of Guangdong Medical College, Rheumatology Institute of Guangdong Medical College, Shenzhen, PR China

${ }^{2}$ Rheumatology Department, Hong Kong University-Shenzhen Hospital, Shenzhen, PR China

"Zhihua Yin and Jinxian Huang contributed equally to this work.

\begin{abstract}
Aim of the study: To investigate the role of 8 cytokines and their correlation with clinical characteristics in systemic lupus erythematosus (SLE) in Han Chinese population by detecting their serum levels using the multiplex fluorescent microsphere method.

Material and methods: Serum was separated from 79 patients with SLE and 40 healthy controls. The serum cytokine detection was conducted according to the instruction of MILLIPLEX MAP human cytokine detection kit on the Luminex liquid phase array platform with $0.01 \mathrm{pg} / \mathrm{ml}$ detectable level. The 8 cytokines were interferon $\alpha 2$ (IFN- $\alpha 2)$, IFN- $\gamma$, interleukin $6(I L-6), I L-8, I L-10, I F N-\gamma$-inducible protein $10(I P-10)$, tumor necrosis factor $\alpha(T N F-\alpha)$ and IL-17. Variable data were in skewed distribution and were expressed with median (P25, P75). Mann-Whitney analysis was used in statistical analysis.

Results: At the baseline level without any stimulus, the level of IP-10 expression was the highest among the 8 cytokines and the second highest was IL-8. The level of IL-17 was too low to be detected. The level of 7 cytokines was higher in SLE patients than in healthy controls $(p<0.01)$. The level of dsDNA antibody, C3, CRP, ESR and anti-nucleosome antibody was correlated with IL-10. Proteinuria was not correlated with any cytokine.

Conclusions: Eight cytokines were measured in our study, while not all of them were detected. The most important finding was the usefulness of IL-10 as a disease activity biomarker for Han Chinese patient with SLE. None of cytokines reflected kidney injury.
\end{abstract}

Key words: systemic lupus erythematosus, cytokine, Han Chinese.

(Centr Eur J Immunol 2014; 39 (2): 228-235)

\section{Introduction}

Systemic lupus erythematosus (SLE) is a common autoimmune disease, and the main feature is activation of autoreactive $\mathrm{T}$ cells and $\mathrm{B}$ cells, which causes production of a large amount of autoantibodies and increase in circulating immune complexes. Up to now, the pathogenesis of autoimmunity in SLE has still not been very clear. There is strong evidence supporting the role of cytokines in the pathogenesis of SLE. Complex interplay between Th1 and Th2-type cytokines is involved and the study showed Th1/ Th2 cytokine profile imbalance existed in SLE patients [1]. Cytokines are soluble factors which are mostly generated by immune cells and in turn play crucial roles in the differentiation, maturation, and activation of various immune cells. Abnormal release or functions of diverse cytokines have been identified in SLE. Some cytokines, including
IL-10, may exert pro- or anti-inflammatory activity, depending on target cell type (e.g. IL-10 inhibits T cell activity but enhances humoral response), hence it is unfavorable in SLE [2].

Currently, the treatment for SLE has evolved from conventional drugs such as corticosteroids and immunosuppressants to biological-target therapies, in which cytokines are the most important therapeutic targets. Knowledge about Tregs and regulatory cytokines would not only provide new insights into the pathogenesis of SLE but could be also used to develop various clinical applications.

The concentrations of 8 cytokines including interferon $\alpha 2$ (IFN- $\alpha 2$ ), IFN- $\gamma$, interleukin 6 (IL-6), IL-8, IL-10, IFN- $\gamma$-inducible protein 10 (IP-10), tumor necrosis factor $\alpha$ (TNF- $\alpha$ ) and IL-17 were measured in sera of SLE patients using multiplex fluorescent microsphere method in

Correspondence: Zhizhong Ye, Nonglin Road 22\#, 518040 Shenzhen, PR China, e-mail: yzh2000@163.com. 
our study. In addition, the relationship between cytokine concentrations and clinical characteristics such as double-stranded DNA (dsDNA) antibody, anti-nucleosome antibody (AnuA), complement components (C3, C4), proteinuria and SLE disease activity index (SLEDAI) [3] was assessed by Spearman's correlation test.

\section{Material and methods}

\section{Subjects}

Seventy-nine patients (76 women and 3 men) aged $34.41 \pm 9.41$ years with SLE were enrolled in our study. The patients were hospitalized in our hospital from 2011 to 2012 . The diagnosis was made according to 1997 American College of Rheumatology guideline. 40 healthy donors ( 35 women and 5 men) aged $37.65 \pm 14.82$ years were recruited from the outpatient clinic, excluding subjects suffering from hypertension, heart disease, diabetes and autoimmune diseases. All the individuals signed the written consent form and the study was approved by the local ethics committee of the hospital.

\section{Clinical features}

Clinical characteristics including dsDNA antibody, AnuA, C3, C4, proteinuria and SLEDAI index were used to assess the disease activity. All patients received standard treatment including mainly steroids and immunosuppressant at different dosage according to their disease activity and degree of organ damage.

\section{Cytokine detection}

Five hundred $\mu \mathrm{l}$ of serum was taken from every individual and stored at $-80^{\circ} \mathrm{C}$. MILLIPLEX MAP human cytokine detection kit was purchased from Millipore Corporation [4]. Measurement of serum cytokine concentrations was conducted according to the instructions. Seven standard samples were set and 2 quality controls, standard sample and detection samples were all assayed in triplicate. The signal value was detected on Luminex chip platform and serum concentration was calculated according to the standard curve. The concentration range was 0-10 000 $\mathrm{pg} / \mathrm{ml}$ for all 8 cytokines and the sensitivity of detection achieved $0.01 \mathrm{pg} / \mathrm{ml}$ level.

\section{Statistical analysis}

Data analysis was conducted by SPSS18.0 software package. For data in skewed distribution, the serum concentration of cytokines was expressed as median (P25, P75). Non-parametric Mann-Whitney U test was used for statisitical analysis. Correlation coefficient was measured using Spearman's correlation test. $P<0.05$ was considered to be statistically significant.

\section{Results}

Demographic and clinical characteristics of patients and controls included in the study are shown in Table 1. The concentration of IP-10 expression was the highest among the 8 cytokines followed by IL- 8 . The concentra-

Table 1. Demographic and clinical characteristics of patients and healthy donors included in the study

\begin{tabular}{|c|c|c|c|}
\hline & $\begin{array}{c}\text { SLE } \\
(n=79)\end{array}$ & $\begin{array}{l}\text { Healthy donors } \\
\qquad(n=40)\end{array}$ & $P$ values \\
\hline Age in years (mean) & $34.41 \pm 9.41$ & $37.65 \pm 14.82$ & 0.212 \\
\hline Female:male & $76: 3$ & $35: 5$ & 0.117 \\
\hline Disease duration (yrs) & $6.91 \pm 6.19$ & - & \\
\hline Age at onset (yrs) & $27.36 \pm 7.67$ & - & \\
\hline ANA $>1: 320$ & $74 / 75$ & - & \\
\hline anti-dsDNA antibody positivity & $32 / 78$ & - & \\
\hline anti-AnuA positivity & $26 / 63$ & - & \\
\hline anti-SmD1 positivity & $39 / 76$ & - & \\
\hline Low C4 & $72 / 79$ & - & \\
\hline Low C3 & $48 / 79$ & - & \\
\hline proteinuria & $22 / 60$ & - & \\
\hline SLEDAI score & $\begin{array}{c}\text { Inactive (0-4): 18/77 } \\
\text { Mild active (5-9): 37/77 } \\
\text { Median active (10-14): 16/77 } \\
\text { Severe active (>14): 6/77 }\end{array}$ & - & \\
\hline
\end{tabular}


Table 2. The serum level of cytokines in patients with SLE and healthy donors expressed as median [(P25, P75), pg/ml]

\begin{tabular}{|c|c|c|c|c|}
\hline \multirow[t]{2}{*}{ Cytokine } & \multicolumn{2}{|c|}{ Group } & \multirow[t]{2}{*}{$Z$ value } & \multirow[t]{2}{*}{$P$ value } \\
\hline & Patients with SLE $(n=79)$ & Healthy donors $(n=40)$ & & \\
\hline IFN- $\alpha 2$ & $0(0,1.28)$ & $0(0,0)$ & -3.417 & 0.001 \\
\hline IFN- $\gamma$ & $0.96(0,2.13)$ & $0(0,0.53)$ & -4.176 & $<0.001$ \\
\hline IL-6 & $0.64(0,4.17)$ & $0(0,0)$ & -4.627 & $<0.001$ \\
\hline IL-8 & $357.99(142.54,934.70)$ & $149.41(73.10,269.96)$ & -3.645 & $<0.001$ \\
\hline IL-10 & $1.19(0,6.05)$ & $0(0,0)$ & -5.594 & $<0.001$ \\
\hline IP-10 & $1191.39(754.73,1979.67)$ & $541.28(446.29,825.53)$ & -5.327 & $<0.001$ \\
\hline TNF- $\alpha$ & $7.97(1.41,15.13)$ & $2.21(0.75,4.45)$ & -4.655 & $<0.001$ \\
\hline IL-17 & $0(0,0)$ & $0(0,0)$ & -1.442 & 0.149 \\
\hline
\end{tabular}

Data are given as medians and $25-75 \%$ range.

tion of IL-17 was too low to be detected. This cytokine was only detected in 7 of 79 SLE patients at the highest concentration of $3 \mathrm{pg} / \mathrm{ml}$ and in one healthy donor at a concentration of $1.26 \mathrm{pg} / \mathrm{ml}$. Relatively low concentrations of IFN- $\alpha 2$, IFN- $\gamma$, IL-6, IL-10 and TNF- $\alpha$ were detected in sera but all of them were higher in SLE patients than that in healthy donors $(p<0.01)$. Similarly, concentrations of IL-8 and IP-10 were higher in SLE patients than in healthy donors $(p<0.01)$ (Table 2). Also IL-17 was correlated with some (IFN- $\gamma$, IL-10 and IL-6) but not all cytokines (Table 3). There was a positive correlation between serum concentrations of the majority of tested cytokines, except IFN- $\alpha 2$ and IL-8.

Among tested cytokines only IL-10 positively correlated with the majority of clinical indices, including SLEDAI, systemic inflammation markers (CRP, ESR), and autoanti- body titres. Consistently, there was also an inverse correlation between this cytokine and serum concentration of $\mathrm{C} 3$, known to be reciprocally associated with the disease activity (Table 4, Fig. 2). Interestingly, also other cytokines (IL-6, TNF, IFN, and IP-10) correlated with systemic inflammation markers (CRP and/or ESR) and the concentration of at least one autoantibody (anti-dsDNA and/or AnuA). By contrast, IL-17 correlated positively only with the concentrations of complement components (C3, C4) (Table 4). However, this finding is uncertain because IL-17 was detected merely in $8.8 \%$ of SLE patients.

Thus, in comparison with healthy volunteers, sera of SLE patients contained significantly higher concentrations of seven cytokines, including IFN- $\alpha 2$, IFN- $\gamma$, IL-6, IL-8, IL-10, IP-10, TNF- $\alpha$ (Fig. 1). Although several cytokines were moderately related to some clinical indices (Table 4),

Table 3. Correlation between concentration of cytokines presented as $r$ and $p$ value

\begin{tabular}{|c|c|c|c|c|c|c|c|c|c|}
\hline & & IFN- $\alpha 2$ & IFN- $\gamma$ & IL-6 & IL-8 & IL-10 & IP-10 & TNF- $\alpha$ & IL-17 \\
\hline \multirow[t]{2}{*}{ IFN- $\alpha 2$} & $r$ & - & 0.048 & 0.071 & -0.033 & 0.065 & 0.091 & 0.163 & -0.048 \\
\hline & $p$ & & 0.674 & 0.532 & 0.770 & 0.566 & 0.425 & 0.152 & 0.672 \\
\hline \multirow[t]{2}{*}{ IFN- $\gamma$} & $r$ & 0.048 & - & 0.664 & 0.131 & 0.331 & 0.332 & 0.663 & 0.291 \\
\hline & $p$ & 0.674 & & 0.000 & 0.250 & 0.003 & 0.003 & 0.000 & 0.009 \\
\hline \multirow[t]{2}{*}{ IL-6 } & $r$ & 0.071 & 0.664 & - & 0.020 & 0.476 & 0.257 & 0.746 & 0.332 \\
\hline & $p$ & 0.532 & 0.000 & & 0.863 & 0.000 & 0.022 & 0.000 & 0.003 \\
\hline \multirow[t]{2}{*}{ IL-8 } & $r$ & -0.033 & 0.131 & 0.020 & - & -0.072 & -0.079 & 0.080 & -0.005 \\
\hline & $p$ & 0.770 & 0.250 & 0.863 & & 0.527 & 0.491 & 0.481 & 0.963 \\
\hline \multirow[t]{2}{*}{ IL-10 } & $r$ & 0.065 & 0.331 & 0.476 & -0.072 & - & 0.390 & 0.457 & 0.249 \\
\hline & $p$ & 0.566 & 0.003 & 0.000 & 0.527 & & 0.000 & 0.000 & 0.027 \\
\hline \multirow[t]{2}{*}{ IP-10 } & $r$ & 0.091 & 0.332 & 0.257 & -0.079 & 0.390 & - & 0.256 & 0.004 \\
\hline & $p$ & 0.425 & 0.003 & 0.022 & 0.491 & 0.000 & & 0.023 & 0.974 \\
\hline \multirow[t]{2}{*}{ TNF- $\alpha$} & $r$ & 0.163 & 0.663 & 0.746 & 0.080 & 0.457 & 0.256 & - & 0.148 \\
\hline & $p$ & 0.152 & 0.000 & 0.000 & 0.481 & 0.000 & 0.023 & & 0.192 \\
\hline \multirow[t]{2}{*}{ IL-17 } & $r$ & -0.048 & 0.291 & 0.332 & -0.005 & 0.249 & 0.004 & 0.148 & - \\
\hline & $p$ & 0.672 & 0.009 & 0.003 & 0.963 & 0.027 & 0.974 & 0.192 & \\
\hline
\end{tabular}


Table 4. Correlation between concentration of cytokines and clinical parameters presented as $r$ and $p$ value

\begin{tabular}{cccccccccc}
\hline & & $\mathbf{C 3}$ & $\mathbf{C 4}$ & CRP & ESR & dsDNA & AnuA & proteinuria & SLEDAI \\
\hline IFN- $\alpha \mathbf{2}$ & $r$ & -0.154 & -0.101 & -0.113 & 0.183 & 0.206 & 0.171 & -0.037 & 0.129 \\
& $p$ & 0.175 & 0.378 & 0.329 & 0.126 & 0.071 & 0.176 & 0.777 & 0.264 \\
\hline IFN- $\gamma$ & $r$ & 0.052 & 0.203 & 0.226 & 0.262 & 0.210 & 0.305 & 0.017 & -0.139 \\
& $p$ & 0.648 & 0.073 & 0.050 & $\mathbf{0 . 0 2 8}$ & 0.065 & $\mathbf{0 . 0 1 4}$ & 0.899 & 0.226 \\
\hline \multirow{2}{*}{ IL-6 } & $r$ & -0.064 & -0.005 & 0.423 & 0.427 & 0.352 & 0.315 & 0.009 & -0.065 \\
& $p$ & 0.578 & 0.965 & $\mathbf{0 . 0 0 0}$ & $\mathbf{0 . 0 0 0}$ & $\mathbf{0 . 0 0 2}$ & $\mathbf{0 . 0 1 1}$ & 0.944 & 0.574 \\
\hline \multirow{2}{*}{ IL-8 } & $r$ & 0.143 & 0.211 & 0.034 & -0.259 & 0.006 & -0.080 & -0.101 & -0.127 \\
& $p$ & 0.208 & 0.062 & 0.769 & $\mathbf{0 . 0 2 9}$ & 0.956 & 0.529 & 0.443 & 0.270 \\
\hline IL-10 & $r$ & -0.271 & -0.221 & 0.373 & 0.532 & 0.298 & 0.457 & 0.241 & 0.333 \\
& $p$ & $\mathbf{0 . 0 1 6}$ & 0.073 & $\mathbf{0 . 0 0 1}$ & $\mathbf{0 . 0 0 0}$ & $\mathbf{0 . 0 0 8}$ & $\mathbf{0 . 0 0 0}$ & 0.064 & $\mathbf{0 . 0 0 3}$ \\
\hline IP-10 & $r$ & -0.157 & -0.195 & 0.207 & 0.387 & 0.141 & 0.424 & -0.029 & 0.141 \\
& $p$ & 0.167 & 0.085 & 0.073 & $\mathbf{0 . 0 0 1}$ & 0.219 & $\mathbf{0 . 0 0 0}$ & 0.828 & 0.221 \\
\hline \multirow{2}{*}{ TNF- $\alpha$} & $r$ & -0.135 & -0.053 & 0.232 & 0.387 & 0.282 & 0.365 & 0.061 & 0.035 \\
& $p$ & 0.236 & 0.642 & $\mathbf{0 . 0 4 4}$ & $\mathbf{0 . 0 0 1}$ & $\mathbf{0 . 0 1 2}$ & $\mathbf{0 . 0 0 3}$ & 0.643 & 0.764 \\
\hline IL-17 & $r$ & 0.234 & 0.222 & 0.180 & 0.088 & 0.154 & 0.015 & -0.049 & -0.112 \\
& $p$ & $\mathbf{0 . 0 3 8}$ & $\mathbf{0 . 0 4 9}$ & 0.120 & 0.463 & 0.179 & 0.905 & 0.712 & 0.333 \\
\hline
\end{tabular}

Data was presented as $(r, p)$ values. $P<0.05$ was considered as statistically significant. Clinical parameters included complements, autoantibodies, inflammation and clinical indices.

IL-10 showed the strongest association, because this cytokine correlated with almost all evaluated parameters (Fig. 2).

\section{Discussion}

Numerous reports have characterised systemic and local cytokine profiles in SLE patients [5-8]. However, the results concerning certain cytokines remain controversial, for example, a significant negative correlation between the levels of IL-10 and SLEDAI scores was found in the northern Indian subjects [9]. Nonetheless, other studies [10-12] reported elevated levels of serum IL-10 in SLE patients which correlated well with SLE disease activity. Several studies have found increased serum levels of IFN- $\gamma$ [13-18] and TNF- $\alpha[14,19-22]$ in patients with active stages of SLE. Other studies have found IFN- $\gamma$ [23-25] and TNF- $\alpha$ [23] levels unaltered in the disease course. Possible explanations of this discrepancy are application of cytokine measurement techniques of different sensitivity, small patients cohort and different ethinity.

Compared with former studies in northern Indian subjects [9], our study might be more convincing due to a larger sample size recruited. Besides, a high resolution approach was applied to detect the cytokines in human specimens in our study. It would be more convenient to detect multiple cytokines simultaneously, while detecting cytokine concentration using ELISA once at a time might probably raise interference. Another advantage was the trifle requirement of serum of only $50 \mu \mathrm{l}$ in our study. Usually, $200 \mu \mathrm{l}$ of serum would be needed in ELISA assay. Therefore, more blood should be taken from patients when detecting several cytokines by ELISA approach.
The results of our study are consistent with some former studies [26-28]. We have found seven cytokines, including IFN- $\alpha 2$, IFN- $\gamma$, IL-6, IL-8, IL-10, IP-10, TNF- $\alpha$ to be overproduced in SLE patients, because serum concentrations of these cytokines were significantly higher than in healthy donors. Despite this, only IL-10 correlated with all disease activity indices.

Numerous studies have demonstrated that serum IL-10 concentration is significantly elevated and correlates with lupus disease activity [10-12]. The correlation was replicated in our Chinese Han population. In murine lupus IL-10 plays a pathologic role, for continuous therapy from the young age with IL-10 antibodies ameliorated autoimmunity in NZB/W F1 mice [29]. In accordance with the therapeutic effect of anti-IL-10 antibodies, the continuous administration of recombinant IL-10 increased disease activity. Similarly, in a small, uncontrolled, open-label study involving patients with mild disease, anti-IL-10 monoclonal antibody improved cutaneous lesions, joint symptoms, and the SLE disease activity index [30].

In addition, IL-10 concentration in the exhaled breath condensate (EBC) and bronchoalveolar lavage fluid (BALF) was higher in patients with SLE compared with healthy donors. Thus, the measurement of IL-10 in the EBC may be a useful biomarker of SLE activity. It is likely that IL-10 protects against pulmonary manifestations of SLE [31].

Although other cytokines present in SLE patients sera in elevated concentrations showed a more limited correlation with clinical indices, they may also contribute to development of various disease symptoms, e.g. lupus nephritis (LN).

The expression of IL- 6 is increased in the kidneys of patients with active lupus nephritis and has been shown 

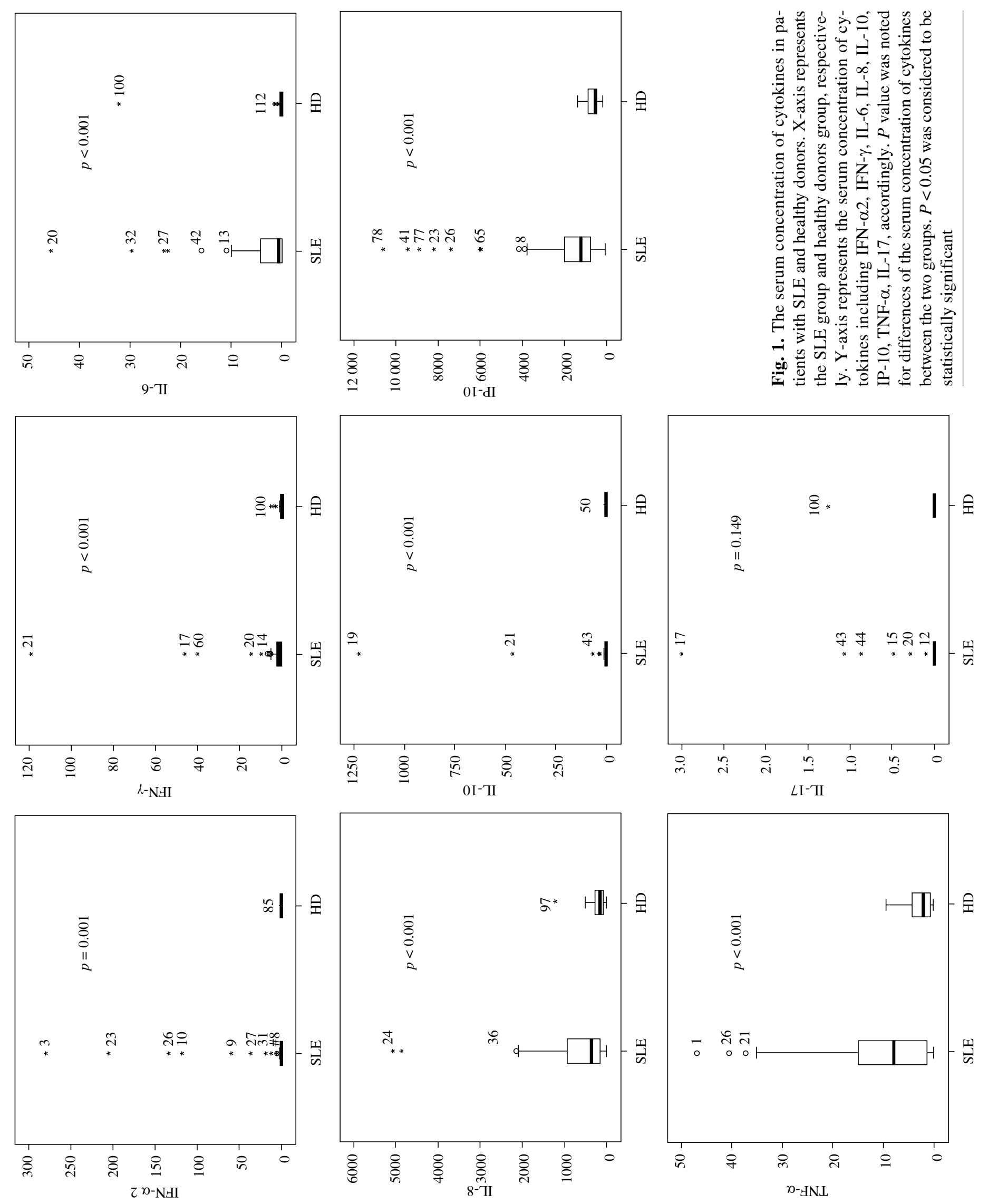

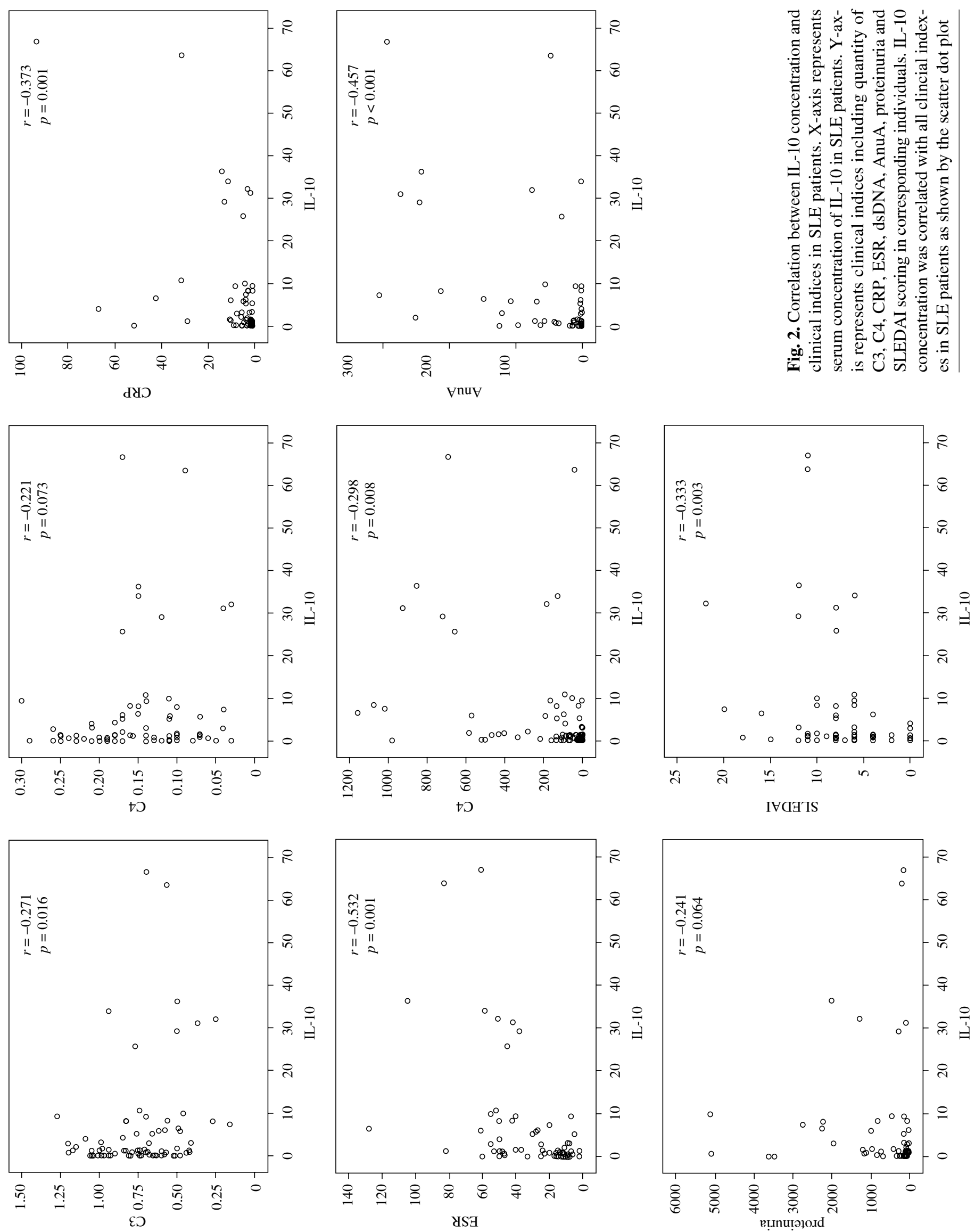
to contribute to disease pathogenesis. Measurement of the IL-6 concentration in SLE patients could help to predict future renal involvement in SLE patients [32].

Numerous studies have defined the cytokine and chemokine profiles in the cerebrospinal fluid (CSF) from patients with NPSLE. Patients with headache had increased CSF concentrations of IL-6, IL-8 and IP-10 compared with non-NPSLE and non-autoimmune diseases patients [5-7].

Patients with severe/extensive skin lesions showed a higher frequency of cytokine gene overexpression. An increased IFN- $\gamma$ expression suggests its involvement in SLE skin inflammation [8].

Recently, the role of IL-23/IL-17 axis in SLE has been studied as well. Significantly higher levels of glomerular IL-17 and IL-23 expression were observed in renal biopsies from class IV LN patients as compared to those from MCN patients and normal controls. Glomerular IL-17 and IL-23 expression levels were positively correlated with renal SLEDAI and histological activity index for LN patients. These results suggest the potential role of the IL-23/ Th17 axis in the intra-renal inflammation of SLE. Serum IL-17 concentration correlates poorly with SLE disease activity, but is significantly elevated in patients with CNS disease [33-35].

Lupus nephritis is one of the most common and serious manifestations of SLE. In our cohort, nearly $55.84 \%$ (43/77) of patients had either previous or current LN. Patients were considered to have active renal disease if proteinuria was above $0.5 \mathrm{mg} / \mathrm{day}$, hematuria was above 5 red blood cells per high-power field, pyuria was above 5 white blood cells/high-power field, or cellular casts were present. Unfortunately, none of tested cytokines is correlated with renal impairment in our study. Compared with kidney tissue biopsy, cytokine from serum source are probably not an ideal approach for assessment of $\mathrm{LN}$ involvement.

IP-10 was repeatedly reported to be correlated with disease activity and clinical manifestations in SLE [36, 37]. However, we failed to show such association in our study. We found this cytokine to be correlated with ESR value and AnuA concentration only (Table 4). This discrepancy may result from distinct population diversity. Further investigation of this cytokine is needed to confirm this supposition.

In conclusion, IL-10 was elevated in Han Chinese patients with SLE and might be used as a serum marker to evaluate disease activity.

The authors declare no conflict of interest.

This research was supported by National grants (81102266, 81301529) and the Shenzhen Key Discipline Foundation (2005C10).

\section{References}

1. Fu Q, Chen X, Cui H, et al. (2008): Association of elevated transcript levels of interferon-inducible chemokines with disease activity and organ damage in systemic lupus erythematosus patients. Arthritis Res Ther 10:R112.

2. Su DL, Lu ZM, Shen MN, et al. (2012): Roles of pro- and anti-inflammatory cytokines in the pathogenesis of SLE. J Biomed Biotechnol 2012: 347141.

3. Bombardier C, Gladman DD, Urowitz MB, et al. (1992): Derivation of the SLEDAI. A disease activity index for lupus patients. The Committee on Prognosis Studies in SLE. Arthritis Rheum 35: 630-640.

4. de Jager W, te Velthuis H, Prakken BJ, et al. (2003): Simultaneous detection of 15 human cytokines in single sample of stimulated peripheral blood mononuclear cells. Clin Diagn Lab Immunol 10: 133-139.

5. Fragoso-Loyo H, Richaud-Patin Y, Orozco-Narváez A, et al. (2007): Interleukin-6 and chemokines in the neuropsychiatric manifestations of systemic lupus erythematosus. Arthritis Rheum 56: 1242-1250.

6. Okamoto H, Iikuni N, Kamitsuji S, et al. (2006): IP-10/MCP1 ratio in CSF is a useful diagnostic marker of neuropsychiatric lupus patients. Rheumatology (Oxford) 45: 232-234.

7. Fragoso-Loyo H, Atisha-Fregoso Y, Llorente L, et al. (2013): Inflammatory profile in cerebrospinal fluid of patients with headache as a manifestation of neuropsychiatric systemic lupus erythematosus. Rheumatology (Oxford) [Epub ahead of print].

8. Carneiro JR, Fuzii HT, Kayser C, et al. (2011): IL-2, IL-5, TNF- $\alpha$ and IFN- $\gamma$ mRNA expression in epidermal keratinocytes of systemic lupus erythematosus skin lesions. Clinics (Sao Paulo) 66: 77-82.

9. Arora V, Verma J, Marwah V, et al. (2012): Cytokine imbalance in systemic lupus erythematosus: a study on northern Indian subjects. Lupus 21: 596-603.

10. Park YB, Lee SK, Kim DS, et al. (1998): Elevated interleukin-10 levels correlated with disease activity in systemic lupus erythematosus. Clin Exp Rheumatol 16: 283-288.

11. Houssiau FA, Lefebvre C, Vanden Berghe M, et al. (1995): Serum interleukin 10 titers in systemic lupus erythematosus reflect disease activity. Lupus 4: 393-395.

12. Koenig KF, Groeschl I, Pesickova SS, et al (2012): Serum cytokine profile in patients with active lupus nephritis. Cytokine 60: 410-416.

13. Chun HY, Chung JW, Kim HA, et al. (2007): Cytokine IL-6 and IL-10 as biomarkers in systemic lupus erythematosus. J Clin Immunol 27: 461-466.

14. Gómez D1, Correa PA, Gómez LM, et al. (2004):Th1/Th2 cytokines in patients with systemic lupus erythematosus: is tumor necrosis factor alpha protective? Semin Arthritis Rheum 33: 404-413.

15. Kim T, Kanayama Y, Negoro N, et al. (1987): Serum levels of interferons in patients with systemic lupus erythematosus. Clin Exp Immunol 70: 562-569.

16. Al-Janadi M, Al-Balla S, Al-Dalaan A, et al. (1993): Cytokine profile in systemic lupus erythematosus, rheumatoid arthritis, and other rheumatic diseases. J Clin Immunol 13: 58-67.

17. Tokano Y, Morimoto S, Kaneko H, et al. (1999): Levels of IL-12 in the sera of patients with systemic lupus erythematosus (SLE) - relation to Th1- and Th2-derived cytokines. Clin Exp Immunol 116: 169-173. 
18. Robak E, Smolewski P, Wozniacka A, et al. (2004): Relationship between peripheral blood dendritic cells and cytokines involved in the pathogenesis of systemic lupus erythematosus. Eur Cytokine Netw 15: 222-230.

19. Davas EM, Tsirogianni A, Kappou I, et al. (1999): Serum IL-6, TNFa, p55 srTNFa, p75 srTNFa, srIL- 2a levels and disease activity in systemic lupus erythematosus. Clin Rheumatol 18: 17-22.

20. Mahmoud RA1, El-Gendi HI, Ahmed HH. (2005): Serum neopterin, tumor necrosis factor-alpha and soluble tumor necrosis factor receptor II (p75) levels and disease activity in Egyptian female patients with systemic lupus erythematosus. Clin Biochem 38: 134-141.

21. Sabry A, Sheashaa H, El-Husseini A, et al. (2006): Proinflammatory cytokines (TNF-alpha and IL-6) in Egyptian patients with SLE: its correlation with disease activity. Cytokine 35 : 148-153.

22. Swadzba J, Iwaniec T, Musial J (2011): Increased level of tumor necrosis factor- $\alpha$ in patients with antiphospholipid syndrome: marker not only of inflammation but also of the prothrombotic state. Rheumatol Int 31: 307-313.

23. Lacki JK, Leszczynski P, Kelemen J, et al. (1997): Cytokine concentration in serum of lupus erythematosus patients: the effect on acute phase response. J Med 28: 99-107.

24. Viallard JF, Pellegrin JL, Ranchin V, et al. (1999): Th1 (IL-2, interferon-gamma (IFN-gamma)) and Th2 (IL-10, IL-4) cytokine production by peripheral blood mononuclear cells (PBMC) from patients with systemic lupus erythematosus (SLE). Clin Exp Immunol: 115: 189-195.

25. Chang DM, Su WL, Chu SJ (2002): The expression and significance of intracellular $\mathrm{T}$ helper cytokines in systemic lupus erythematosus. Immunol Invest 31: 1-12.

26. Brugos B, Vincze Z, Sipka S, et al. (2012): Serum and urinary cytokine levels of SLE patients. Pharmazie 67: 411-413.

27. Koenig KF, Groeschl I, Pesickova SS, et al. (2012): Serum cytokine profile in patients with active lupus nephritis. Cytokine 60: 410-416.

28. McCarthy EM, Smith S, Lee RZ, et al. (2014): The association of cytokines with disease activity and damage scores in systemic lupus erythematosus patients. Rheumatology (Oxford) [Epub ahead of print].

29. Ishida H, Muchamuel T, Sakaguchi S, et al. (1994): Continuous administration of anti-interleukin 10 antibodies delays onset of autoimmunity in NZB/W F1 mice. J Exp Med 179: 305-310.

30. Ravirajan CT, Wang Y, Matis LA, et al. (2004): Effect of neutralizing antibodies to IL-10 and C5 on the renal damage caused by a pathogenic human anti-dsDNA antibody. Rheumatology (Oxford) 43: 442-447.

31. Nielepkowicz-Goździńska A, Fendler W, Robak E, et al. (2013): Exhaled cytokines in systemic lupus erythematosus with lung involvement. Pol Arch Med Wewn 123: 141-148.

32. Yung S, Cheung KF, Zhang Q, et al. (2013): Mediators of inflammation and their effect on resident renal cells: implications in lupus nephritis. Clin Dev Immunol 2013: 317682.

33. Vincent FB, Northcott M, Hoi A, et al. (2013): Clinical associations of serum interleukin-17 in systemic lupus erythematosus. Arthritis Res Ther 15: R97.

34. Ambrosi A, Espinosa A, Wahren-Herlenius M (2012): IL-17: a new actor in IFN-driven systemic autoimmune diseases. Eur J Immunol 42: 2274-2284.
35. Chen DY, Chen YM, Wen MC, et al. (2012): The potential role of Th17 cells and Th17-related cytokines in the pathogenesis of lupus nephritis. Lupus 21: 1385-1396.

36. Okamoto H, Iikuni N, Kamitsuji S, et al. (2006): IP-10/MCP-1 ratio in CSF is an useful diagnostic marker of neuropsychiatric lupus patients. Rheumatology (Oxford) 45: 232-234.

37. Kong KO, Tan AW, Thong BY, et al. (2009): Enhanced expression of interferon-inducible protein-10 correlates with disease activity and clinical manifestations in systemic lupus erythematosus. Clin Exp Immunol 156: 134-140. 